\title{
Comparison of the Effects of Different Organ Preservation Surgeries on Voice Quality by Perceptual and Acoustic Methods
}

\author{
Fatma Esen Aydınlı1 (D), Ahmet Ataş² (D), Şefik Hoşal ${ }^{3}$ (i) \\ 1Department of Speech and Language Therapy, Hacettepe University School of Health Sciences, Ankara, Turkey \\ ${ }^{2}$ Department of Audiology, i̇stanbul University School of Health Sciences, İstanbul, Turkey \\ ${ }^{3}$ Center of Otorhinolaryngology, Ankara, Turkey
}

Cite this article as: Esen Aydınlı F, Ataş A, Hoşal Ş. Comparison of the Effects of Different Organ Preservation Surgeries on Voice Quality by Perceptual and Acoustic Methods. JAREM 2019; 9(1): 14-21.

\begin{abstract}
Objective: As a result of partial or total surgical removal of the larynx due to larynx cancer, there are several aspects of patient's life that are altered, such as the anatomical, physiological, psychological, and social aspects. One of the key elements that affect the quality of life of postoperative patients in organ-preserving surgeries is the vocal function.

This study is designed as a prospective case-controlled study aimed at comparing the degree of dysphonia occurring after the implementation of different conservational laryngeal surgery types. The voice quality of the study group $(n=49)$ consisting of the individuals who underwent endolaryngeal laser surgery, vertical partial laryngectomy, supraglottic laryngectomy, and supracricoid partial laryngectomy has been compared with the voice quality of healthy control group $(n=20)$ individuals.

Methods: After receiving the patient history, evaluations were made, including perceptual analysis, videolaryngoscopic examination, and acoustic analysis. For all evaluations, the control group and the study group findings were compared.

Results: According to the perceptual and acoustic analysis scores, the supraglottic laryngectomy subjects had the closest findings to the normal voice quality, whereas the supracricoid partial laryngectomy group was the most distant among surgery groups. There were no significant differences between the endolaryngeal laser surgery and vertical partial laryngectomy participants. The supraglottic compression parameter was found to be worse in supracricoid partial laryngectomy compared to supraglottic laryngectomy and endolaryngeal laser surgery.

Conclusion: Although the findings of the acoustic analysis and perceptual voice evaluation results obtained in this study are generally consistent with the literature, the present study differs in that it compared four different partial surgical procedures with a versatile voice evaluation. Limitations to this study are not considering different reconstruction techniques and no subgroups equal in number.
\end{abstract}

Keywords: Conservational laryngeal surgery, larynx cancer, voice evaluation, voice quality

\section{INTRODUCTION}

Laryngeal cancer can be treated by radiotherapy, surgery, chemotherapy, or a combination of these treatments. The partial or complete removal of the larynx as a result of the surgical treatment changes the anatomical, physiological, psychological, and social factors in the patient (1). The effects of these changes on the voice production mechanism of patients are also observed. Patients often complain of hoarseness, diminished volume, increased vocal effort, or breathe vocal production (2).

In the determination of the most appropriate treatment option for laryngeal cancer, many factors, such as the length of the tumor, as well as the pretreatment laryngeal function, concomitant chronic diseases, and patient expectations, should be considered (3-7). Organ preservation surgery, which is one of the treatment options, is defined as a combination of surgeries in which part of the larynx is removed; thus, physiological functions, such as speech, swallowing, and respiration, are maintained $(7,8)$. Endolaryngeal laser surgery (ELC), supraglottic laryngectomy (SGL), vertical partial laryngectomy (VPL), and supracricoid partial laryngectomy (SCPL) are common techniques used among these surgeries $(7,9)$. Although vocal functions are of great importance in the patients' quality of life after laryngeal surgery, there are limited data in the literature about vocal function results protected by different surgical techniques $(10,11)$. In a limited number of studies comparing the different types of organ preservation sur- 
gery, voice quality has not been evaluated in a wide range with subjective and objective aspects $(12,13)$.

Voice handicap indexes, acoustic analysis, and perceptual analysis are the most commonly used methods to evaluate voice quality (14). In acoustic analysis, fundamental frequency (F0), jitter, shimmer, and noise/harmonic ratio (NHR) are more commonly measured (15-17). Perceptual analysis, which is accepted under the heading of subjective analysis evaluation, is the method in which the voice is evaluated by the individuals working in the field of voice (speech and language therapists and/or laryngologists) and evaluated by the human ear $(9,18,19)$. Although there are other methods aimed at the same objective, the GRBAS method is accepted as the gold standard method in the literature $(11,20)$. In the GRBAS method, G (grade), voice quality with all its features; $R$ (roughness), irregular fluctuations in voice and roughness; $B$ (breathiness), turbulence created by air leak; A (astheny), weakness in voice, weakness, and hypokinetic (hypofunctionality); and S (strain), refers to excessive exertion, tension, or hyperfunctional (hyperkinetic) pitch are assessed. In this method, the degree of voice disturbance is determined by giving a value between 0 and 3 points for each of the five expressions (20).

Various methods are used in the instrumental evaluation of voice quality. Videolaryngostroboscopy (VLS), which is among these methods, aids to visualize the structural changes and abnormal movement of the larynx $(21,22)$.

In the literature, there are many data related to acoustic analysis and perceptual analysis findings in the patient group undergoing organ preservation surgery, whereas data on VLS assessment findings are limited $(23,24)$.

Since voice is multidimensional, it must be examined objectively and subjectively $(14,18,25)$. However, there are different results in the correlations between the results of perceptual analysis and acoustic analysis findings obtained from different studies (24). Therefore, the use of multiple modalities is of particular importance when evaluating the degree of voice impact in patients undergoing organ preservation surgery $(14,24)$.

The main purpose of the present study was to evaluate the degree of distortion of the voice quality of patients treated with different organ preservation surgeries due to laryngeal cancer. Such an evaluation would provide useful information to experts in the selection of the most appropriate surgical technique for the treatment of tumors with varying degrees of local progression, with regard to the impact on voice quality.

\section{METHODS}

The evaluations of the study and control groups included in our study were performed in the Department of Otolaryngology and Audiology and Speech Disorders Department of Hacettepe University School of Medicine (Ethics committee decision no.: LUT 10/27-8). Written informed consent was obtained from patients who participated in this study.

\section{Individuals}

The study group consisted of 60 male participants who underwent partial laryngeal surgery, and the control group consisted of 20 male participants with normal otorhinolaryngology (ENT) results. The voice recordings of 60 participants in the study group were examined according to Vans-Brooks' criteria, and the findings of 49 patients who were judged to be able to perform acoustic voice analysis were evaluated (26).

Inclusion criteria of the individuals in the study group were no relapse and no metastasis of tumor in the last ENT examination, date of evaluation at least 2 months after the date of laryngeal surgery, and no accompanying systematic or neurological disease. Inclusion criteria of the control group were no complaint in any period, no systematic or neurological disease, no history of upper respiratory tract infection that may affect the voice quality on the evaluation day, not using any drug, not smoking, and normal hearing.

\section{Voice Assessment}

\section{Acoustic Evaluation}

Acoustic measurement was performed using the Computerized Speech Lab Model 4300B-Kay Elemetrics. Each individual involved in the study was informed by the clinician prior to recording as to what he/she was required to do before enrollment and to repeat at least once according to the condition of the individual. During the recording, the individual was asked to stand upright, and the microphone was recorded at a distance of $15 \mathrm{~cm}$ from the left side at an angle of $45^{\circ}$ (28). Subjects were guided to maintain the same pitch and loudness during recording; the la/ voice was recorded for at least $5 \mathrm{~s}$ via the Multidimensional Voice Profile (MDVP). The analysis of the records obtained from acoustic measurements was made according to the criteria proposed by Vans-Brooks in the acoustic analysis of alaryngeal voice using a narrow band (600 dot) spectrogram (26). Eleven records that were not considered to be suitable for acoustic analysis were excluded from the study. In MDVP analysis, the first and last seconds of recording were subtracted, and the middle 3-second interval was obtained (27). In MDVP analysis, F0, jitter, shimmer, NHR, Sound Turbulence Index, and Soft Phonation Index (YFI) parameters were taken into consideration.

\section{Perceptual Evaluation}

\section{Equipment}

Individuals were asked to read a 117-word standard text in "The Last Birds" in the normal pitch and loudness for the GRBAS assessment. Recordings were made using the Philips Brand SA3115/02 model, frequency response between 80 and $18 \mathrm{kHz}$, NHR Pulse $80 \mathrm{~dB}$ greater than mono, with a built-in microphone Adaptive Differential Pulse-Code Modulation voice recording program that contains the voice recorder. The records were stored in Wav file format.

\section{Assessment}

The evaluation was made by two Audiology and Speech-Language Pathology Specialists who have at least 5 years of experience in the field of voice who listened to the voice recordings twice at different times. The scoring was graded as follows: 0: normal, 1: slightly affected, 2: moderately affected, and 3: severe effects. The results were interpreted by calculating the average of the assessment scores of the two specialists. 


\section{Videolaryngostroboscopy Evaluation}

\section{Equipment}

The evaluation was performed using the light source of KAY PENTAX RLS 9100B in the Department of ENT using the 9200 C Digital Strobe. A rigid endoscope was used in the evaluation, and a local anesthetic agent (lidocaine) was used in cases where the participant had too much retention.

\section{Evaluation}

The images were recorded with the patient's name after the evaluation, and the analyses were performed by an experienced ENT specialist in the field of larynx surgery according to a standard evaluation form. The reviewers were blinded to the study. In this form, the glottal closure parameter was scored as follows: 1: normal, 2: intermittent, and 3: incomplete; mucosal wave existence parameter was scored as follows: 1: normal, 2: less/no, and 3: increased; supraglottic compression parameter was scored as follows: 1: none and 2-5: severe; amplitude parameter was scored as follows: 1: normal and 2-5: fix; phase and asymmetry parameter was scored as follows: 1 : irregularity none and 2-5:

\section{Table 1. Age characteristics of the individuals included in the study according to the groups}

\begin{tabular}{|c|c|c|c|c|}
\hline \multirow[b]{2}{*}{ Group } & \multirow[b]{2}{*}{$\mathrm{n}$} & \multicolumn{3}{|c|}{ Age (year) } \\
\hline & & Min. & Ave & Max. \\
\hline Control & 20 & 41 & 49.85 & 78 \\
\hline Study & 49 & 41 & 56.53 & 73 \\
\hline
\end{tabular}

Table 2. Intraobserver reliability coefficient of GRBAS parameters

\begin{tabular}{|l|c|c|c|c|c|}
\hline Observers & G & R & B & A & S \\
\hline $1^{\text {st }}$ observer & 0.818 & 0.652 & 0.833 & 0.72 & 0.716 \\
\hline $2^{\text {nd }}$ observer & 0.854 & 0.722 & 0.799 & 0.782 & 0.854 \\
\hline
\end{tabular}

Table 3. Interobserver reliability coefficient of GRBAS parameters

\begin{tabular}{|l|c|c|c|c|c|}
\hline Value & G & R & B & A & S \\
\hline Cronbach's Alpha & 0.929 & 0.827 & 0.895 & 0.883 & 0.923 \\
\hline
\end{tabular}

always irregular; vocal fold limit parameter was scored as follows: 1: straight and 2-5: rough; inactive segment existence parameter was scored as follows: 1: yes and 0: no (22).

\section{Statistical Analysis}

Statistical Package for Social Sciences version 15.0 package program (SPSS Inc., Chicago, IL, USA) was used for statistical analysis. Numerical variables were represented as mean, standard deviation, median, and minimum and maximum values. Qualitative variables were represented as number and percentage (\%). Kruskal-Wallis test was used to determine whether there was any difference between the groups with regard to numerical variables. Bonferroni-corrected Mann-Whitney test was used for multiple comparisons in case of differences between the groups. Chi-square test was used to examine the difference between the groups with regard to qualitative variables. Spearman correlation coefficients were used to determine the relationship between numerical variables. Cronbach's alpha coefficient was used in the reliability analysis of GRBAS evaluation. A $p$ value $<0.05$ was considered significant in four group comparisons, and a $p$ value $<0.008$ was considered significant in Bonferroni-corrected tests.

\section{Results}

\section{Demographic Findings}

The study included all male participants. The age of the participants was 41-78 years. Table 1 shows the age characteristics of the individuals included in the study.

The study group consisted of 49 participants between the ages of 41 and 73 years with a mean age of 56.53 years. The control group consisted of 20 participants between the ages of 41 and 78 years with a mean age of 49.85 years.

Of the 49 subjects in the study group, 13 were VPL, 12 were SGL, 12 were SCPL, and 12 were ELC.

The time interval between the postoperative evaluations of the participants included in the study was at least 2 months and 165.47 months at most, with a median value of 20 months. Two of the participants in the study group underwent web excision surgery, and no patient received voice therapy.

\section{Evaluation Findings}

\section{Perceptual Analysis Findings}

\section{Reliability}

Cronbach's alpha coefficient was used in the reliability analysis of GRBAS evaluation. The interobserver and intraobserver analysis

Table 4. Distribution of GRBAS median values by the type of surgery

\begin{tabular}{|l|c|c|c|c|c|}
\hline Surgical type & G & R & B & A & S \\
\hline ELC & Moderate & Mild-moderate & Mild-moderate & Mild & Mild \\
\hline VPL & Moderate & Moderate & Moderate & Mild-moderate & Moderate \\
\hline SGL & Mild-moderate & Mild & Mild-moderate & Mild & Mild \\
\hline SCPL & Moderate-severe & Moderate-severe & Moderate-severe & Mild-moderate & Mild-moderate \\
\hline
\end{tabular}

$0=$ normal, $1=$ mild, $1-2=$ mild-moderate, $2=$ moderate, $2-3=$ moderate-severe, $3=$ severe 
results provided reliability for all values above the recommended values in the literature. The intraobserver reliability coefficients are shown in Table 2, and the interobserver reliability coefficient values are shown in Table 3.

In the intraobserver analysis, it was seen that both observers had the lowest reliability values in parameter $\mathrm{R}$. The highest reliability was observed in parameter $G$ for the first observer and in parameters $\mathrm{G}$ and $\mathrm{S}$ for the second observer.

The reliability coefficient from the largest to the smallest was determined as $G, S, B, A$, and $R$ in the interobserver analysis.

The Kruskal-Wallis test $(p<0.05)$ found a difference between the types of surgery with regard to GRBAS evaluation scores, and the Mann-Whitney $U$ test was used to compare the two groups. A p value $<0.008$ was considered significant. Table 4 shows the distribution of GRBAS influences of the groups by considering the median values for each parameter.

According to Table 4, the groups with minimal perceptions of sound quality are SGL and ELC; the most affected groups are VPL and SCPL. There was a significant difference between the VPL and SGL groups in all parameters of GRBAS $(p<0.008)$. GRBAS values were higher in the VPL patient group. A significant difference was found between the ELC and VPL groups only in parameter $S(p<0.008)$. In the VPL group, the scores of parameter $S$ were found to be higher. There was a significant difference between the SGL and SCPL groups in all parameters except for parameter $A(p<0.008)$. The values of the SCPL group were higher in parameters $G, R, B$, and $S$.

To summarize the other findings, no significant difference was found between the ELC and SGL groups with regard to any of the parameters. No significant difference was found between the

Table 5. F0 values in the study and control groups

\begin{tabular}{|l|c|}
\hline Surgical type & F0 (Hz) \\
\hline Study group & \\
\hline Endolaryngeal laser surgery & 169.00 \\
\hline Vertical partial laryngectomy & 184.00 \\
\hline Supraglottic laryngectomy & 156.50 \\
\hline Supracricoid partial laryngectomy & 217.50 \\
\hline Control group & 133.12 \\
\hline
\end{tabular}

ELC and SCPL groups in any of the parameters. No significant difference was found between the VPL and SCPL groups with regard to any of the parameters.

\section{Acoustic Analysis Results}

Higher FO values were observed in the SGL, VPL, ELC, and SCPL groups compared with the control group, but they were not statistically significant ( $p>0.005)$. Table 5 shows the FO values in the patient and control groups.

In the study group, the highest FO value was found in the SCPL group, and the lowest FO was found in the SGL group. In the study group, if the FO values are sorted from the highest to the lowest, they can be listed as SCPL, VPL, ELC, and SGL.

The values obtained from the jitter, shimmer, NHR, STI, and YFI parameters of the control group were found to be different from the values obtained in the VPL, SCPL, and ELC groups for the same parameters $(p<0.008)$.

Jitter, shimmer, NHR, and STI parameters were significantly different between the control group and the SGL group $(p<0.008)$.

In the jitter parameter, it was observed that the SGL group had lower median values among the surgical types. Median values from the lowest to the highest were determined in the control, SGL, VPL, ELC, and SCPL groups.

In the shimmer parameter, the lowest median value among the surgical types belongs to the SGL group. SGL is followed by ELC; the values obtained in the VPL and SCPL groups are similar. There was a statistically significant difference between SGL and SCPL surgery types in jitter, shimmer, NHR, and YFI parameters. The numerical values of these parameters were higher in the SCPL group. There was a significant difference between VPL and $S G L$ in the shimmer parameter $(p<0.008)$. The values of the two parameters were found to be higher in the VPL group.

\section{VLS Analysis Findings}

In the glottal closure parameter, the median glottal closure parameter score of all groups was found to be $\geq 2$, but there was no statistically significant difference between the groups. The presence of mucosal wave, phase and asymmetry, amplitude, and vocal boundary parameters was observed to be affected in patients, and it was seen that still segments were present in each type of surgery; however, no statistically significant difference was found between the types of surgery. The presence of mucosal wave could not be evaluated in two individuals.

Table 6. Amplitude evaluation of the right and left vocal folds according to the types of surgery

\begin{tabular}{|c|c|c|c|c|c|c|c|c|c|c|}
\hline \multirow{3}{*}{ Surgical type } & \multicolumn{10}{|c|}{ Amplitude } \\
\hline & \multicolumn{5}{|c|}{ Right vocal fold } & \multicolumn{5}{|c|}{ Left vocal fold } \\
\hline & $\mathrm{N}$ & F2 & F3 & $\mathrm{F} 4$ & F5 & $\mathrm{N}$ & $\mathrm{F} 2$ & F3 & F4 & F5 \\
\hline ELC & 4 & 2 & 0 & 2 & 3 & 1 & 2 & 0 & 3 & 6 \\
\hline VPL & 5 & 1 & 2 & 2 & 0 & 2 & 2 & 3 & 0 & 4 \\
\hline SGL & 3 & 1 & 5 & 1 & 2 & 3 & 2 & 0 & 2 & 5 \\
\hline
\end{tabular}


Table 7. Indication of glottal closure and mucosal wave characteristics by the type of surgery

\begin{tabular}{|c|c|c|c|c|c|c|c|}
\hline \multirow[b]{3}{*}{ Surgical type } & \multirow{2}{*}{\multicolumn{3}{|c|}{ Glottal closing }} & \multicolumn{4}{|c|}{ Mucosal wave } \\
\hline & & & & \multicolumn{2}{|c|}{ Right } & \multicolumn{2}{|c|}{ Left } \\
\hline & Incomplete & Complete & Intermittent & Normal & Decreased & Normal & Decreased \\
\hline ELC & 7 & 1 & 4 & 4 & 8 & 2 & 10 \\
\hline VPL & 5 & 2 & 5 & 4 & 8 & 8 & 4 \\
\hline SGL & 6 & 1 & 6 & 5 & 7 & 3 & 9 \\
\hline SCPL & 5 & 1 & 6 & 1 & 11 & 1 & 11 \\
\hline
\end{tabular}

The amplitude, symmetry, and vocal fold parameters of the SCPL group could not be evaluated because the anatomical features of surgery did not match. All measurements for these parameters were calculated between the other three types of surgery. The amplitude results are shown in Table 6.

The amplitude of the vocal fold motion was basically examined as normal amplitude and fixed vocal fold, and if the fixed option was chosen, it was scored within $2^{\circ}-5^{\circ}$. The distribution of glottal closure and mucosal wave parameters according to the types of surgery is shown in Table 7.

As shown in Table 7, the mucosal wave was evaluated separately for the right and left vocal folds. In the VPL group, the mucosal wave was decreased on the side of the resection (right/left).

Supraglottic compression parameters differed between the groups in the evaluation of VLS. In summary, there was a significant difference between the ELC and SCPL groups ( $p=0.004)$, and the SCPL group score was higher. There was a significant difference between the SGL and SCPL groups $(p=0.003)$, and the SCPL group scores were higher.

\section{DISCUSSION}

In healthy subjects, while the main part of the larynx, which serves as the vibration, is the vocal folds, after partial laryngectomy, different regions of the larynx serve as a vibrating oscillator. Since anatomy and physiology are affected by varying degrees in these surgeries, it is important to evaluate the sound quality after surgery. In our study, moderate to severe effects on parameters $G, R$, and $B$ and mild to moderate effects of parameters $A$ and $S$ were observed in the SCPL group. In the studies by Lallemant et al. (29), Bron et al. (30), and Makeieff et al. (31), GRBAS scores were moderate to severe in parameters $R$ and $G$, mild to moderate in parameter B, mild in parameter A, and mild or moderate in parameter $\mathrm{S}$. The most affected parameters in the studies by Bron (30) and Makeieff (31) were determined as R, B, S, and A, and the authors stated that there are no interobserver and intraobserver reliability measurements. In our study, the most affected parameters in the SCPL patient group were $G, R, B, A$, and $S$, respectively. In the present study, interobserver and intraobserver reliability measurements were made, and the rates were rather high. The findings show that in SCPL, the perceptual effects on the overall sound quality and the coarseness in the sound are evident.

When the VLS findings in the literature were examined with regard to SCPL, Torrejano et al. (32) reported the vibrating parts of the neo-larynx as the anterior and upper mucosal surfaces of the arytenoids, the lower part of the tongue root, and sometimes the lateral walls of the hypopharynx via the VLS evaluation. Weinstein et al. (33) reported that the mucosal wave in patients with SCPL who underwent the cricohyoid epiglottopexy (CRP) method is in the flexible mucosa of the arytenoid cartilages, but mainly between the arytenoid cartilages and the rigid surface of the epiglottis.

It should be emphasized here that there are many parameters that we have evaluated in our study, such as glottal closure, symmetry, and mucosal wave, in the VLS examination of the larynx (22), although most of these cannot be used in patients with partial laryngectomy. Some researchers have developed different forms of VLS for use in the evaluation of different surgeries (33). The purpose of our study was to compare different types of surgery with the same evaluation methods; thus, the standard VLS form was used. However, vocal fold, amplitude, and symmetry parameters were excluded from the parameters related to vocal folds that could not be evaluated anatomically in this patient group, and the mucosal wave parameter could not be evaluated in some patients. In other studies in the literature, Weinstein et al. (33) stated that the number of protected arytenoid does not affect the sound quality, and that the deteriorated sound quality can be attributed to the inability of the glottal closure. In our study, supraglottic compression parameters that were examined in the VLS evaluation in the SCPL patient group were found to be rather high, and there was a significant difference between the ELC and SGL groups. The possible causes of this difference can be considered as uncontrolled and excessive supraglottic compensation activity to ensure adequate vibration. The presence of mucosal wave in the VLS evaluation of patients with SCPL was found to be decreased/absent in $>90 \%$ of the patients.

If the results of the acoustic analysis of the SCPL group were examined, FO value was found to be $217.50 \mathrm{~Hz}$ in our study. In the literature, while the FO value was reduced in the studies by Weinstein and Webster $(33,34)$, the F0 value was increased in the study by Kuauhyama et al. (35) where the FO value was found to be $243.7 \mathrm{~Hz}$.

In our study, all parameters except F0, which was examined in the MDVP program for the evaluation of acoustic analysis, were different in the SCPL group from the control group. It was also found that the SCPL group was worse with regard to the shimmer and jitter values than the SGL group. However, individuals with SCPL in our study were not investigated in two separate groups as SCP and CRP. 
When similar studies in the literature are investigated, in the study by Lacourreye et al. (36), jitter, shimmer, and NHR were higher in the SCPL group, which is in accordance with our results. Pastore et al. (37) found that all acoustic parameters except intensity are found to be different from the control group. The jitter and shimmer values were also increased in the studies by Kuauhyama et al. (35) and Heather et al. (2).

The VPL among the study groups was the group with the highest value after SCPL according to GRBAS scores. In the literature, there are many studies that support dysphonia as a result of glottic insufficiency after VPL; however, to our knowledge, no study has been found on GRBAS parameters $(2,7,10)$. In VLS findings, there were insufficient glottal closure in the VLS, irregular mucosal waves, decrease in amplitude, abnormal arytenoid movements during vocal fold adduction, and laryngeal edema and erythema in four patients (24).

In our study, the VLS evaluation of the VPL group revealed that the glottal closure parameter was incomplete in five patients, interrupted in five patients, and complete in two patients. The mucosal wave and amplitude parameters of the other parameters decreased on VPL surgery.

In our study, the F0 value of individuals with VPL was $184.00 \mathrm{~Hz}$. This value was not statistically different compared with the control group, but it was numerically high. Kim et al. (10) found that the FO value is higher in the control group. The possible reasons suggested were the shortening of the vibrating area, increased stiffness, and decreased mass effect. However, in the study by Hirano (38), F0 was found to be lower in the control group.

In our study, the sound quality of VPL patients in acoustic analysis was rather different from the control group that was observed by the differences in the jitter, shimmer, NHR, STI, and YFI values. There is a significant difference between the VPL group and the SGL group with regard to the shimmer value. This result indicates that only the intensity irregularities among the two groups are lower in the SGL group.

In the literature, difference was detected in the shimmer and jitter parameters in the VPL group with the control group, which is in accordance with our study (24). The jitter and shimmer parameters generally reflect the vocal fold stability, and the asymmetry of the vocal folds also prevents air flow (24). It is thought that the excessive noise caused by incomplete glottal closure is a factor that increases the jitter and shimmer parameters $(7,39)$.

In the VLS evaluations of the ELC group, one patient with complete glottal closure, four patients with discontinuity, and seven patients with incomplete closure were found. There was no statistically significant tendency with regard to the mucosal wave and amplitude parameters. The ELC group values of the supraglottic compression parameter were found to be less than the SCPL group values. These findings suggest that even if the glottic closure in the ELC group is not complete, an effective phonation can be performed.

In our study, a significant difference was found in all parameters except F0 when compared with the control group. This finding suggests a significant deterioration of the acoustic signal after the ELC. However, no difference was observed with the other sur- gical groups in the acoustic analysis. This may be related to the fact that individuals in the ELC group have varying degrees of resection depth.

Ledda et al. (40) found the percentage of glottal closure to be high in patients with limited excision in the surface layer of the lamina propria or in the Reinke's cavity and/or vocal ligament. They found that the medial part of the vocalis muscle, the internal perichondrium of the thyroid cartilage, the additional vocal fold, and the percentage of the glottal closure in the resection including the arytenoid, ventricular fold, or subglottic region are lower. In this study, the VLS, GRBAS, and MDVP findings of the two groups of patients compared with the depth of resection indicated that the sound quality of the first group was close to that of normal subjects, and that there were significant changes in the sound in the other groups (deeper resection).

According to the GRBAS results, the SGL group is the group with the best sound quality. When the results of the VLS evaluation were considered in this patient group, there were one patient with complete glottal closure and six patients with discontinuous and incomplete closure. In the VLS evaluation, the only parameter that differed between the groups was supraglottic compression. There was a significant difference between SGL and SCPL. This finding suggests that individuals with SCPL use the vocal folds in phonation and therefore need less compensation. In our study, it could be expected that the glottal closure rate of VLS parameters could be higher because the vocal folds were preserved in this surgery, but the scores for this parameter were not different from the other groups in VLS findings.

In SGL, resection usually involves the removal of the entire epiglottis, false vocal folds, aryepiglottic folds, pre-epiglottic space, and upper half of the thyroid cartilage. As reported by Wein et al. (41), the sound quality of the larynx portion responsible for phonation is the best in this type of surgery after the vocal folds are preserved. There is almost normal sound. However, Calcaterra et al. (42) stated that owing to the discontinuation of nervous laryngeal superior to the cricothyroid muscle, the upper pitches cannot be reached, and that the voice can sound "aspirated" due to the inability to clear the secretions in the oropharynx and the protective functions of the epiglottis and aryepiglottic fold. Sparano et al. (9) reported that another effect of surgery is that resection of the supraglottic larynx often leads to a change in the resonance characteristics of the voice.

In our study, the difference was found in the jitter, shimmer, NHR, and STI parameters during the acoustic analysis of the group undergoing SGL surgery. The fact that there is no difference in YFI values compared with other types of surgery suggests that the closure power of the vocal folds is better in this group. However, the difference in jitter parameter indicates the irregular closure and asymmetric vibrations in vocal folds, differences in the shimmer values indicate irregularities regarding loudness, and NHR parameter still indicates the presence of loudness in the voice. The results of the perceptual analysis and acoustic analysis obtained in our study show that near-normal sound is obtained in the patient group undergoing SGL surgery, but there are many distortions in the voice signal. 
According to the findings by Kim et al. (10), jitter and shimmer parameters were seen to be worsening, and it was emphasized that only the resonance cavity narrowed with regard to voice quality.

\section{CONCLUSION}

The results of acoustic analysis and perceptual sound assessment obtained in the present study are generally in accordance with the literature; it differs from other studies in that it compares the four different types of partial surgery simultaneously with a voice evaluation. The most important limitation of the study is the lack of an equal number of subgroups by considering other features, such as different reconstruction techniques.

In further studies, the study of the effect of different surgeries on the sound may make it easier for us to understand the mechanisms that affect voice quality by making more homogeneous groups including other factors, such as surgical reconstruction, resection depth, and/or radiotherapy.

Ethics Committee Approval: Ethics committee approval was received for this study from the Ethics Committee of Hacettepe University (Approval number: LUT 10/27-8).

Informed Consent: Written informed consent was obtained from patients who participated in this study.

Peer-review: Externally peer-reviewed.

Author Contributions: Concept - A.A.; Design - A.A.; Supervision A.A., Ş.H.; Resources - A.A., Ş.H.; Data Collection and/or Processing Ş.H., F.E.A.; Analysis and/or Interpretation - A.A., Ş.H., F.E.A.; Literature Search - A.A., F.E.A.; Writing Manuscript - F.E.A., A.A.; Critical Review - A.A., Ş.H.

Conflict of Interest: The authors have no conflict of interest to declare.

Financial Disclosure: The authors declared that this study has received no financial support.

\section{REFERENCES}

1. Batıoğlu Karaaltın A, Binbay Z, Yiğit Ö, Dönmez Z. Evaluation of life quality, self-confidence and sexual functions in patients with total and partial laryngectomy. Auris Nasus Larynx 2017; 44: 188-94. [CrossRef]

2. Heather M, Starmer MA, Donna C, Tippett MPH, Kimberly T, Webster MA. Effects of Laryngeal Cancer on Voice and Swallowing. Otolaryngol Clin North Am 2008; 41: 793-818. [CrossRef]

3. Laccourreye OLL, Garcia D, Guttierez-Fonseca R, Brasnu D, Weinstein G. Vertical partial laryngectomy versus supracricoid laryngectomy for selected carcinomas of the true vocal cord classified as T2N0. An Otol Rhinol Laryngol 2000; 109: 965-71. [CrossRef]

4. Dinescu FV, Tiple C, Chirila M, Muresan R, Drugan T, Cosgarea M. Evaluation of health-related quality of life with EORTC QLQ-C30 and QLQ-H\&N35 in Romanian laryngeal cancer patients. Eur Arch Otorhinolaryngol 2016; 273: 2735-40. [CrossRef]

5. Forastiere AA, Weber RS, Trotti A. Organ preservation for advanced larynx cancer: issues and outcomes. J Clin Oncol 2015; 33: 3262-68. [CrossRef]

6. Sadoughi B. Quality of life after conservation surgery for laryngeal cancer. Otolaryngol Clin North Am 2015; 48: 655-65. [CrossRef]

7. Chawla S, Carney AS. Organ preservation surgery for laryngeal cancer. Head Neck Oncol 2009; 1: 12. [CrossRef]

8. Shumrick DA. Conservation Surgery of the Larynx. Cincinnati J Med 1968; 49: 1.

9. Sparano A, Ruiz C,Weinstein GS. Voice rehabilitation after external partial laryngeal surgery. Otolaryngol Clin North Am 2004; 37: 63753. [CrossRef]
10. Kim C, Lim YC, Kim K, Kim Y, Choi HS, Kim KM, et al. Vocal Analysis After Vertical Partial Laryngectomy. Yonsei Med J 2003; 44: 1034-9. [CrossRef]

11. Sittel C, Eckel HE, Eschenburg C. Phonatory results after laser surgery for glottic carcinoma. J Otolaryngol Head Neck Surg 1998; 119: 418-24. [CrossRef]

12. Peetres A, Van Gogh C, Goor K. Health status and voice outcome after treatment for T1 a glottic carcinoma. Eur Arch Otorhinolaryngol 2004; 261: 534-40. [CrossRef]

13. Kandogan T, Sanal A. Quality of life, functional outcome, and voice handicap index in partial laryngectomy patients for early glottic cancer. BMC Ear Nose Throat Disord 2005; 5: 3. [CrossRef]

14. Khildr A. Ramos C. Bless DM. Heisy D. Resolving the battle between internal and external standards for visual perceptual ratings of laryngeal images:an essential step towards reliable research protocol. Meeting of the ASHA; 1997 November, Boston Massachustes; 1997.

15. Kent BD, Ball MJ. Voice Quality Measurement, San Diego: Singular Publishing Group; 2000.

16. Zhang Y, Jiang JJ. Acoustic Analyses of Sustained and Running Voices From Patients With Laryngeal Pathologies. J Voice 2008: 22; 1-9. [CrossRef]

17. Maryn Y, Corthals P, De Bodt M, Van Cauwenberge P, Deliyski D. Perturbation Measures of Voice:A Comparative Study between Multi-Dimensional Voice Program and Praat. Folia Phoniatr Logop 2009; 61: 217-26. [CrossRef]

18. Wolfe V, Fitch J, Cornell R. Acoustic prediction of severity in commonly occurring voice problems. J Speech Hear Res 1995; 38: 273-9. [CrossRef]

19. Bele IV. Reliability in perceptual analysis. J Voice 2005; 19: 555-73. [CrossRef]

20. Webb AL, Carding PN, Deary IJ. The realibility of three perceptual evaluation scales for dysphonia. Eur Arch Otorhinolaryngol 2003; 261: 429-34

21. Poburka BJ. A New Stroboscopy Rating Form. J Voice 1999; 13: 40313. [CrossRef]

22. Wendler J. Stroboscopy. J Voice 1999; 2: 149-54.

23. Kazi R, Venkitaraman R, Johnson C. EEG comparision of voice outcomes in patientns with advanced laryngopharyngeal cancer treated by chemoradiotheraphy or total laryngectomy. Int J Radiat Oncol Biol Phys 2008; 70: 344-52. [CrossRef]

24. Kazi R, Singh A. Multidimensional Assessment of Voice After Vertical Partial Laryngectomy: A Comparison With Normal and Total Laryngectomy Voice. J Voice 2008; 22: 740-5. [CrossRef]

25. Yücetürk $A V$, Günhan K. Multidimensional assessment of voice and speech after supracricoid laryngectomy. J Laryngol Otol 2004; 118 : 791-95. [CrossRef]

26. vanAs-Brooks CJ, Koopmans-van Beinum FJ, Pols LC, Hilgers FJ.Acoustic signal typing for evaluation of voice quality in tracheoesophageal speech. J Voice 2006; 20: 355-68. [CrossRef]

27. Kent RD, Vorperian HK, Duffy JR. Reliability of the Multi-Dimensional Voice Program for the analysis of voice samples of subjects with dysarthria. Am J Speech Lang Pathol 1999; 8: 129-136. [CrossRef]

28. Yu P, Ouaknine M, Revis J, Giovanni A. Objective Voice Analysis for Dysphonic Patients: A Multiparametric Protocol Including Acoustic and Aerodynamic Measurements. J Voice 2001; 15: 52942. [CrossRef]

29. Lallemant JG, Bonnin P, El-Sioufi I, Bousquet J. Cricohyoepiglottopexy: Long-term results in 55 patients. J Laryngol Otol 1999; 113: 532. [CrossRef]

30. Bron L, Pasche P, Brossard E, Schweizer V. Functional analysis after supracricoid partial laryngectomy with cricohyoidoepiglottopexy. Laryngoscope 2002; 112: 1289-93. [CrossRef] 
31. Makeieff M, Barbotte E, Giovanni A, Guerrier B. Acoustic and aerodynamic measurement of speech production after supracricoid partial laryngectomy. Laryngoscope 2005; 115: 546-51. [CrossRef]

32. Torrejano G, Guimaraes I. Voice Quality after supracricoid laryngectomy and Total laryngectomy with insertion of voice prosthesis. J Voice 2007; 23: 240-6. [CrossRef]

33. Weinstein GS, Laccourreye O, Ruiz C, Dooley P. Larynx preservation with supracricoid partial laryngectomey with cricohyoidoepiglottopexy. Ann Otol Rhinol Laryngol 2002; 111: 1. [CrossRef]

34. Webster KT, Samlan RA, Jones B, Bunton K, Tufano RP. Supracricoid partial laryngectomy: swallowing, voice and speech outcomes. Ann Otol Rhinol Laryngol 2010; 119: 10-6. [CrossRef]

35. Kuahuyuma, Nunez-Valencia ER, Tamez-Velarde M, Granados-Garcia M. Quality of life and functional evaluation after supracricoid partial laryngectomy with cricohyoidoepiglottopexy in Mexican patients. J Laryngol Otol 2004; 118: 284-8.

36. Laccourreye O, Crevier-Buchmann L, Weinstein G, Biacabe B, Laccourreye $H$, Brasnu D. Duration and frequency characteristics of spe- ech and voice following supracricoid partial laryngectomy. Ann Otol Rhinol Laryngol 1995; 104: 516-21. [CrossRef]

37. Pastore A1, Yuceturk AV, Trevisi P. Evaluation of voice and speech following subtotal reconstructive laryngectomy. Eur Arch Otorhinolaryngol 1998; 255: 371-4. [CrossRef]

38. Hirano M, Kurita S, Matsuoka H. Vocal function following hemilaryngectomy. Ann Otol Rhinol Laryngol 1987; 96: 586-9. [CrossRef]

39. Tufano RP. Open supraglottıc laryngectomy. Oper techn otolaryngol head neck surg 2003; 14: 22-6. [CrossRef]

40. Ledda GP, Grover N, Pudvir V. Functional Outcomes after CO2 Laser treatment of early glottic carcinoma. Laryngoscope 2006; 16: 1007 11. [CrossRef]

41. Wein RO, Weber RS. The current role of vertical partial laryngectomy and open supraglottic laryngectomy. Curr Probl Cancer 2005; 29: 201-14. [CrossRef]

42. Calcaterra TC, Zwitman DH. Vocal Rehabilitation after Partial or Total Laryngectomy. Calif Med 1972; 117: 12-5. 